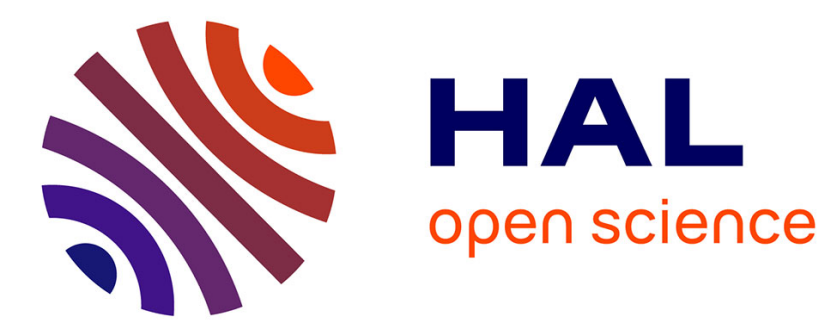

\title{
A review of magnetostatic moment method
}

\author{
Olivier Chadebec, Jean-Louis Coulomb, Fleur Janet
}

\section{To cite this version:}

Olivier Chadebec, Jean-Louis Coulomb, Fleur Janet. A review of magnetostatic moment method. IEEE Transactions on Magnetics, 2006, 42 (4), pp.515 à 520. 10.1109/TMAG.2006.870929 . hal00189512

\section{HAL Id: hal-00189512 \\ https://hal.science/hal-00189512}

Submitted on 25 Jun 2008

HAL is a multi-disciplinary open access archive for the deposit and dissemination of scientific research documents, whether they are published or not. The documents may come from teaching and research institutions in France or abroad, or from public or private research centers.
L'archive ouverte pluridisciplinaire HAL, est destinée au dépôt et à la diffusion de documents scientifiques de niveau recherche, publiés ou non, émanant des établissements d'enseignement et de recherche français ou étrangers, des laboratoires publics ou privés. 


\title{
A Review of Magnetostatic Moment Method
}

\author{
Olivier Chadebec $^{1}$, Jean-Louis Coulomb ${ }^{1}$, and Fleur Janet ${ }^{2}$ \\ ${ }^{1}$ Laboratoire d'Electrotechnique de Grenoble (LEG-CNRS/INPG/UJF) LEG/ENSIEG, 38402 Grenoble, France \\ ${ }^{2}$ Schneider Electric, Corporate Research Division, 38050 Grenoble, France
}

\begin{abstract}
This paper proposes a review of the magnetostatic moments method (MoM) applied to model electromagnetic devices. This method is now well-known for its "light weight" and its simplicity of implementation. Its main advantages are the nonrequirement of an air region mesh and a coarse mesh of the ferromagnetic material. It leads to very fast resolution and very accurate field, force, and moment computations. The paper proposes a state of the art of this approach and shows some efficient realizations.
\end{abstract}

Index Terms-Magnetization identification, magnetostatic moment method, point-matching approach, simplified moment method.

\section{INTRODUCTION}

$\mathbf{M}$ AGNETOSTATIC moments method (magnetostatic MoM) is now known as a powerful approach to model electrotechnical devices. Developed more than 30 years ago by Harrington [1], several academic codes like GFUN [2], RADIA [3], or CALMAG3D [4] have already shown the reliability of the approach. Nowadays, it can be an interesting alternative to classical finite-element methods (FEM) in some situations.

In this method, only ferromagnetic material parts are divided into elementary elements with a uniform magnetization associated with each of them. These elements are called moments and the distribution naturally respects Maxwell's equations. The main idea of the approach is that the magnetic field is created by the inductor sources and all the moments defined in the problem. With a point matching technique, which ensures the validity of the materials constitutive laws, a system is obtained. It remains to solve it to obtain the values of moments and then to compute the magnetic field everywhere. Force or flux can thus be easily computed too.

The main advantage of the approach is that air (or equivalent) does not require any mesh. Only ferromagnetic materials and magnets are meshed and often with coarse subdivisions. It leads to very high-speed resolutions and to high accuracies for stray field computations, in comparison with FEM. Most part of references found in the literature deals with this use of moment method. However, other interesting applications can be found.

The method could be called a "light weight" approach because of its simplicity of implementation, its speed, and above all its efficiency to solve complex problems with very few numbers of unknowns. It is why this method is particularly well adapted for multistatic studies with motion or optimization strategy (no mesh of the air region is required at each step and the evaluation of a problem is very fast). Finally, the method can easily be inverted and so gives very good results for magnetization identification.

However, to apply moment method to all these applications is not without difficulties. The use of this approach needs a good knowledge of the physical phenomena and of the numerical method itself. In opposition to FEM, which is very general, the moment method requires a high level of how-to skill.

This paper proposes a review of this method based on significant applications. First, we will sum up the main concepts of the theory. Linear and nonlinear models will be considered. Different kinds of elements will be shown in order to reduce the computation times and increase the accuracy of the method. Moreover, we will show some numerical realizations.

\section{BASIC THEORY}

\section{A. Standard Equation}

Let us consider a magnetostatic problem composed by some ferromagnetic regions and coils in which currents flow. Equations governing the problem are

$$
\begin{aligned}
\operatorname{div} \mathbf{B} & =0 \\
\operatorname{curl} \mathbf{H} & =\mathbf{J} .
\end{aligned}
$$

We must add the classical following material law [5]:

$$
\mathbf{M}_{\text {ind }}-\mathbf{M}_{\text {rem }}=f(\mathbf{H}) \mathbf{H}
$$

where $\mathbf{M}_{\mathbf{i n d}}$ is called the induced magnetization of the material and $\mathbf{M}_{\mathbf{r e m}}$ is the permanent one. It is usual to divide the magnetic field $\mathbf{H}$ into the summation of two terms: $\mathbf{H}_{0}$, the source magnetic field created by currents and $\mathbf{H}_{\text {red }}$, the reduced magnetic field created by the ferromagnetic material. As we have

$$
\operatorname{curl} \mathbf{H}_{0}=\mathbf{J}
$$

we can conclude that the reduced magnetic field derives from a scalar potential

$$
\mathbf{H}(\mathrm{P})=\mathbf{H}_{\mathbf{0}}(\mathrm{P})-\operatorname{grad} \varphi_{\text {red }}(\mathrm{P})
$$

where $\mathrm{P}$ is a point located everywhere in the domain region. The reduced scalar potential is equal to [6]

$$
\varphi(\mathrm{P})=\frac{1}{4 \pi} \iiint_{\mathrm{V}} \frac{\left(\mathbf{M}_{\text {ind }}+\mathbf{M}_{\mathbf{r e m}}\right) \cdot \mathbf{r}}{\mathbf{r}^{3}} \mathrm{dV}
$$

where $\mathrm{V}$ is the volume of ferromagnetic material and $\mathbf{r}$ the vector between the integration point and the point $\mathrm{P}$ where the 


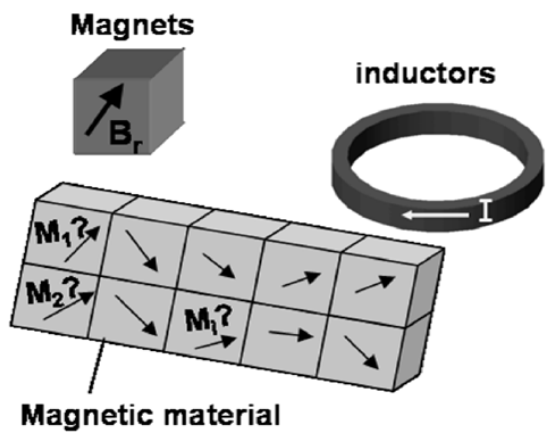

Fig. 1. Device under study, with magnets, inductors, and ferromagnetic parts meshed into volume elements.

field is expressed. Let us notice that the expression (6) ensures the validity of (1). By calculating the gradient of (6), we get thus an equation for the magnetic field

$$
\begin{aligned}
\mathbf{H}(\mathrm{P})= & \mathbf{H}_{0}(\mathrm{P})+\frac{1}{4 \pi} \iiint_{\mathrm{V}}\left(\frac{3\left(\mathbf{M}_{\mathbf{i n d}} \cdot \mathbf{r}\right)}{\mathrm{r}^{5}} \mathbf{r}-\frac{1}{\mathrm{r}^{3}} \mathbf{M}_{\mathbf{i n d}}\right) \mathrm{dV} \\
& +\frac{1}{4 \pi} \iint_{\text {Vmagnet }} \int_{\text {in }}\left(\frac{3\left(\mathbf{M}_{\mathbf{r e m}} \cdot \mathbf{r}\right)}{\mathrm{r}^{5}} \mathbf{r}-\frac{1}{\mathrm{r}^{3}} \mathbf{M}_{\text {rem }}\right) \mathrm{dV} .
\end{aligned}
$$

In the integral terms, readers will recognize the standard expression of field created by a dipoles volume distribution.

\section{B. Mesh of the Device}

Let us consider a device composed of several magnets ( $\mathrm{V}_{\text {magnet }}$ ) with a known magnetization $\mathbf{M}_{\mathbf{r e m}}$, inductors in which flows static currents I and ferromagnetic parts (V) with unknown magnetization $\mathbf{M}_{\text {ind }}$ (see Fig. 1).

The ferromagnetic material is meshed into $\mathrm{J}$ volume elements and the magnets are divided into $\mathrm{K}$ volume elements too. Let us assume that magnetizations are uniform in each element of the ferromagnetic material and in the magnets. Equation (7) becomes

$$
\begin{aligned}
& \mathbf{H}(\mathrm{P})=\mathbf{H}_{0}(\mathrm{P})+\frac{1}{4 \pi} \sum_{\mathbf{j}=1}^{\mathrm{J}} \iint_{\mathrm{V}_{\mathbf{j}}} \int\left(\frac{3\left(\mathbf{M}_{\mathbf{i n d}_{-} \mathbf{j}} \cdot \mathbf{r}\right)}{\mathrm{r}^{5}} \mathbf{r}-\frac{1}{\mathrm{r}^{3}} \mathbf{M}_{\mathbf{i n d}_{-\mathbf{j}}}\right) d \mathrm{~V}_{\mathbf{j}} \\
& +\frac{1}{4 \pi} \sum_{\mathrm{k}=1}^{\mathrm{K}} \int_{\mathrm{V}_{\text {magnet_k }}} \iint\left(\frac{3\left(\mathbf{M}_{\mathbf{r e m} \_\mathbf{k}} \cdot \mathbf{r}\right)}{\mathrm{r}^{5}} \mathbf{r}-\frac{1}{\mathrm{r}^{3}} \mathbf{M}_{\mathbf{r e m} \_\mathbf{k}}\right) \mathrm{d} \mathrm{V}_{\mathrm{k}}
\end{aligned}
$$

Now, let us define an orthogonal basis in each volume element for the magnetization. As (8) is linear, it can be rewritten as it follows:

$$
\mathbf{H}(\mathrm{P})=\mathbf{H}_{\mathbf{0}}(\mathrm{P})+\{\mathbf{g}\}\left[\mathbf{m}_{\mathbf{r e m}}\right]+\{\mathbf{f}\}\left[\mathbf{m}_{\mathbf{i n d}}\right]
$$

where coefficients $\mathbf{m}_{\mathbf{i n d}}$ and $\mathbf{m}_{\mathbf{r e m}}$ define magnetizations in each local basis, $\{\mathbf{g}\}$ is a $(3 \times 3 \mathrm{~K})$ matrix, $\{\mathbf{f}\}$ is a $(3 \times 3 \mathbf{J})$ matrix. Let us notice that $\left[\mathbf{m}_{\mathbf{r e m}}\right]$ is a $(3 K \times 1)$ vector and $\left[\mathbf{m}_{\mathbf{i n d}}\right]$ is a $(3 \mathrm{~J} \times 1)$ one.

\section{Point-Matching Method}

A simple way to obtain an approximate solution of (7) is to ensure that this equation will be satisfied at discrete points in the region of interest. This procedure is called point matching approach. In our method, chosen points will be the barycenter of each element $\mathrm{i}$ of ferromagnetic regions. Equation (9) will be written at each barycenter of each element and projected in its local basis.

1) Linear Material Law: In the case of a linear ferromagnetic material, the relation between the magnetization and the field is

$$
\mathbf{M}_{\text {ind }}=\left(\mu_{r}-1\right) \mathbf{H}
$$

By combining (9) and (10), we can write a linear system

$$
\left\{\left\{\mathbf{I}_{\mathbf{d}}\right\}-\{\mathbf{F}\}\right\}\left[\mathbf{m}_{\text {ind }}\right]=\left[\mathbf{h}_{\mathbf{0}}\right]+\{\mathbf{G}\}\left[\mathbf{m}_{\mathbf{r e m}}\right]
$$

with $3 \times \mathrm{J}$ unknowns. $\mathbf{I}_{\mathbf{d}}$ is the identity matrix, and $\left[\mathbf{h}_{\mathbf{0}}\right]$ the source field at each barycenter projected in local basis. It can easily be computed by analytical or numerical computation of Biot and Savart's law. Let us notice that in this model, (1) and (2) are satisfied by construction. In fact, the moment method solves (10) (i.e., the material law) by a numerical approximation (i.e., a point matching approach).

The system obtained is square and full. This is the main drawback of the approach. In fact, for large meshes, the matrix of this system become too memory consuming and required too much time to be solved. It is the most important drawback of the method in comparison with the finite-element one.

2) Nonlinear Law: For a nonlinear material, an iterative algorithm is required (relaxation) using the (3). In some cases, the convergence is difficult to reach, but some now well-known techniques can ensure and improve the convergence speed [7].

\section{INTEGRATION IMPROVEMENTS}

\section{A. Reduction of the Singularity}

The main difficulty in moment method is the computation of matrix $\mathbf{F}$ coefficients (and/or $\mathbf{G}$ matrix too). If we compute the influence of element $\mathrm{j}$ at the barycenter of element $\mathrm{i}$, the coefficient will have the following expression:

$$
\mathrm{f}_{\mathrm{ij}}=\left\langle\left(\frac{1}{4 \pi} \iiint_{\mathrm{V}_{\mathrm{j}}}\left(\frac{3\left(\mathbf{b}_{\mathbf{j}} \cdot \mathbf{r}_{\mathrm{ij}}\right)}{\mathrm{r}_{\mathrm{ij}}^{5}} \mathbf{r}_{\mathrm{ij}}-\frac{1}{\mathrm{r}_{\mathrm{ij}}^{3}} \mathbf{b}_{\mathbf{j}}\right) \mathrm{dV} \mathbf{j}\right), \mathbf{b}_{\mathbf{i}}\right\rangle
$$

where $\langle$,$\rangle denotes the classical scalar product operator. Let us$ notice that $(12)$ is a $(3 \times 3)$ matrix for volume element. In particular, for $\mathrm{i}=\mathrm{j}$ (computation of the influence of an element on itself), even if this integral is analytically convergent, the use of numerical integration can lead to artificial numerical singularity (i.e., Gauss integration points are too close from the matching point). Moreover for an unspecified element shapes, no analytical solution for (12) can be found.

A well-known solution is to consider that all the elements are spherical (or better ellipsoidal). Equation (12) has thus a very simple expression in all the cases [8]. However, if this technique can be acceptable to compute the field created by magnet in air, it can lead to important inaccuracies when ferromagnetic materials are taken into account.

The best solution is to use the well-known equivalent surface charge distribution. As we said, the magnetization $\mathbf{M}_{\mathbf{i n d}}$ is constant on each element. Its divergence is equal to zero on the volume. On this assumption, the field created by the volume 


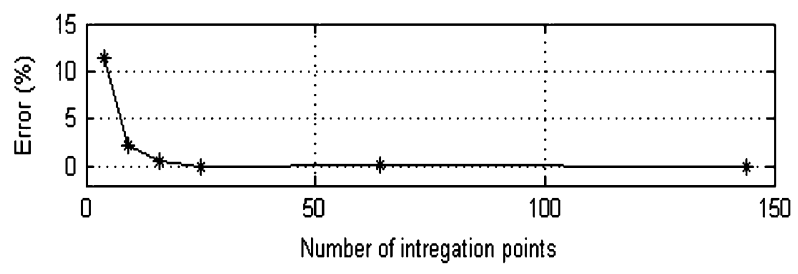

Fig. 2. Computation of the influence of a cube volume element on itself (point-matching at the element barycenter). The magnetization is parallel to a face. Curve shows values of (13) obtain for different number of Gauss points and compared with analytical solution.

is exactly the same that the field created by a surface charge distribution $\mathbf{M}_{\mathbf{i n d}} \cdot \mathbf{n}$ located on the surface delimiting the volume [6] (the vector $\mathbf{n}$ is the external normal of $\mathrm{V}$ ). The advantage of this approach is to present a reduced singularity $\left(\sim 1 / \mathrm{r}^{2}\right)$ in comparison with (12) $\left(\sim 1 / \mathrm{r}^{3}\right)$. Equation (12) becomes thus

$$
\mathrm{f}_{\mathrm{ij}}=\left\langle\left(\frac{1}{4 \pi} \iint_{\mathrm{Si}} \mathbf{b}_{\mathbf{j}} \cdot \mathbf{n}_{\mathbf{j}} \frac{\mathbf{r}_{\mathrm{ij}}}{\mathrm{r}_{\mathrm{ij}}^{3}} \mathrm{dS} \mathrm{S}_{\mathrm{i}}\right), \mathbf{b}_{\mathbf{i}}\right\rangle .
$$

The integral can be computed analytically [7], [9], so with a great accuracy. However, formulas are complex to implement and their evaluation can increase the computation time. It is thus possible to use numerical Gauss technique. It allows us to use a vectorized algorithm ("for" loops are replaced by matrix manipulations). Computation time decreases, but the accuracy decreases too.

As it is shown in Fig. 2, an important number of integration points (16 for a square surface) is needed to ensure an error inferior to $0.5 \%$. This number still leads to high computation time even if a vectorized algorithm is used. Our preferred solution is to use a limited number of integration points (four per rectangular faces for example) and to recompute matrix terms which represent the influence of an element on itself with an analytical integration. It allows us to increase the speed of the algorithm, the accuracy remaining very good.

\section{B. Other Elements}

For some particular geometries, the direction of magnetization in the device can be known. Equation (13) is thus simplified and the number of unknowns is significantly reduced. In this section, we present two different kinds of elements: surface one, which allows to model shells, and line ones dedicated to rods modelings.

1) Surface Elements: Let us consider a ferromagnetic shell. We consider that the permeability of the sheet is high and that the thickness e is small in comparison with other dimensions. The field in the sheet is mainly tangential and constant through the thickness of the shell [10]. Equation (13) becomes then

$$
\mathrm{f}_{\mathrm{ij}}=\left\langle\left(\frac{\mathrm{e}}{4 \pi} \iint_{\mathrm{Si}} \mathbf{b}_{\mathbf{j}} \cdot \mathbf{n}_{\mathbf{j}} \frac{\mathbf{r}_{\mathrm{ij}}}{\mathrm{r}_{\mathrm{ij}}^{3}} \mathrm{dL}\right), \mathbf{b}_{\mathbf{i}}\right\rangle .
$$

The shell needs thus to be meshed only by surface elements (elements are not meshed in the thickness). Charges are then located on segments delimiting the surface elements. Surface integration becomes thus line ones and only two bases $\left(b_{1}, b_{2}\right)$ tangential to the surface element are used ( $\mathbf{n}$ is the external normal of the element and is tangential to it). It allows us to reduce significantly the number of unknowns. Analytical and numerical

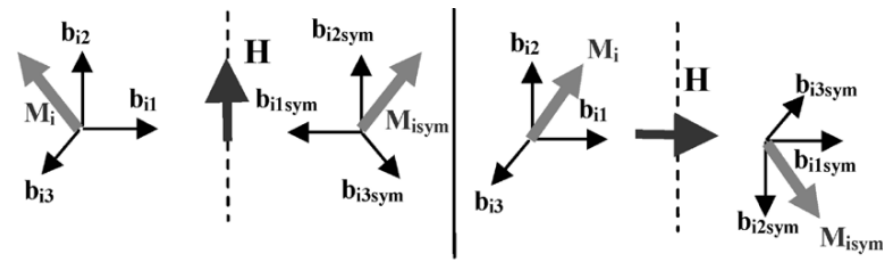

(a)

(b)

Fig. 3. Bases description to take into account symmetrical problems. Two cases are represented: (a) tangential symmetry, and (b) normal symmetry for the magnetic field.

integrations can be provided. Reference [11] compares obtained results.

2) Line Elements: Let us now consider a device composed of ferromagnetic rods (small section $\mathrm{s}$ and high permeability). By applying the same approach that in previous section, we can model the rod by segments (line elements) with charges located at the extremity of each of them. Equation (13) becomes

$$
f_{i j}=\left\langle\left(\frac{s}{4 \pi} \sum_{1,2} \mathbf{b}_{\mathbf{j}} \cdot \mathbf{n}_{\mathbf{j}} \frac{\mathbf{r}_{i j}}{r_{i j}^{3}}\right), \mathbf{b}_{\mathbf{i}}\right\rangle .
$$

Only one basis is need ( $b_{1}$ tangential to the line element). In some particular case, an high mesh density is necessary to take into account important variation of the magnetization along the rod. The length of elements may become so small that the section of the rod cannot be neglected any more. Special integration, taken into account the shape of the section can thus be provided [12].

\section{SYMMETRIES}

The main drawback of the magnetostatic MoM is that linear systems obtained are full. Therefore, the memory needed to store and solved them increased dramatically with the number of element $\left(\mathrm{n}^{3}\right)$. It is thus absolutely necessary to develop a tool which allows to take into account geometrical and physical symmetries of the device.

Let us represent a main basis $\left(\mathbf{b}_{\mathbf{i} 1}, \mathbf{b}_{\mathbf{i} \mathbf{2}}, \mathbf{b}_{\mathbf{i} \mathbf{3}}\right)$ of volume element number $\mathbf{i}$ (with a magnetization $\mathbf{M}_{\mathbf{i}}$ ) and its symmetrical elements (with a magnetization $\mathbf{M}_{\mathbf{i s y m}}$ ) with an associated basis $\left(\mathbf{b}_{\mathbf{1 i s y m}}, \mathbf{b}_{\mathbf{2 i s y m}}, \mathbf{b}_{\mathbf{3 i s y m}}\right)$. This symmetrical basis will depend on the symmetry of the field in the device (see Fig. 3).

Let us write $\mathbf{M}_{\mathbf{i}}$ and $\mathbf{M}_{\mathbf{i s y m}}$ in their associated bases. In both cases (a) and (b), coefficient $\mathrm{m}_{\mathrm{i} 1,2,3}$ obtained are exactly the same to describe the magnetization associated to element $i$ and its symmetrical magnetization

$$
\begin{aligned}
\mathbf{M}_{\mathbf{i}} & =\mathrm{m}_{\mathrm{i} 1} \mathbf{b}_{\mathbf{i} 1}+\mathrm{m}_{\mathrm{i} 2} \mathbf{b}_{\mathbf{i} 2}+\mathrm{m}_{\mathrm{i} 3} \mathbf{b}_{\mathbf{i} \mathbf{3}} \\
\mathbf{M}_{\mathbf{i s y m}} & =\mathrm{m}_{\mathrm{i} 1} \mathbf{b}_{\mathbf{i} 1 \mathbf{s y m}}+\mathrm{m}_{\mathrm{i} 2} \mathbf{b}_{\mathbf{i} 2 \mathbf{s y m}}+\mathrm{m}_{\mathrm{i} 3} \mathbf{b}_{\mathbf{i} 3 \mathbf{3 s y m}} .
\end{aligned}
$$

Now, we write the global system obtained without taking into account symmetries. To simplify expressions, the permanent magnetization is not taken into account. Thanks to (11), we get the following linear system:

$$
\begin{array}{r}
\left\{\begin{array}{cc}
\left.\{\mathbf{F}\}+\left\{\mathbf{I}_{\mathbf{d}}\right\}\right\}_{\mathrm{m} \rightarrow \mathrm{m}} & \{\mathbf{F}\}_{\mathrm{sym} \rightarrow \mathrm{m}} \\
\{\mathbf{F}\}_{\mathrm{m} \rightarrow \mathrm{sym}} & \left\{\{\mathbf{F}\}+\left\{\mathbf{I}_{\mathbf{d}}\right\}\right\}_{\mathrm{sym} \rightarrow \text { sym }}
\end{array}\right\}\left[\begin{array}{c}
\mathrm{m}_{\mathrm{m}} \\
\mathrm{m}_{\mathrm{sym}}
\end{array}\right] \\
=\left[\begin{array}{c}
\mathrm{h}_{\mathbf{0}} \\
\mathrm{h}_{\mathbf{0} \mathrm{sym}}
\end{array}\right] .
\end{array}
$$


With our bases, we can notice that

$$
\begin{aligned}
\left\{\{\mathbf{F}\}+\left\{\mathbf{I}_{\mathbf{d}}\right\}\right\}_{\mathrm{m} \rightarrow \mathrm{m}} & =\left\{\{\mathbf{F}\}+\left\{\mathbf{I}_{\mathbf{d}}\right\}\right\}_{\mathrm{sym} \rightarrow \mathrm{sym}} \\
\{\mathbf{F}\}_{\mathrm{m} \rightarrow \text { sym }} & =\{\mathbf{F}\}_{\mathrm{sym} \rightarrow \mathrm{m}} \\
\mathrm{h}_{\mathbf{0}} & =\mathrm{h}_{\mathbf{\text { sym }}} \\
\mathrm{m}_{\mathbf{m}} & =\mathrm{m}_{\text {sym }} .
\end{aligned}
$$

It remains to write a new linear system which takes into account symmetries of the problem

$$
\left\{\left\{\{\mathbf{F}\}+\left\{\mathbf{I}_{\mathbf{d}}\right\}\right\}_{\mathrm{m} \rightarrow \mathrm{m}}+\{\mathbf{F}\}_{\mathrm{sym} \rightarrow \mathrm{m}}\right\}\left[\mathbf{m}_{\mathbf{m}}\right]=\left[\mathbf{h}_{\mathbf{0}}\right] .
$$

Let us consider that the main region has n volume elements, the symmetrical one has thus $n$ elements too. The computation of matrix (23) leads to $n \times 2 n$ operations against $2 n \times 2 n$. The integration time is then divided per 2 . Moreover, the space memory needed to store the matrix is $3 \times n \times 3 \times n$ against $3 \times 2 n \times 3 \times 2 n$. It is then reduced per 4 . The computation time to solve the system is then divided per 8 .

\section{Post-Processing}

\section{A. Stray Field Computation}

The stray field created by the device can easily be computed with (8). The main advantage of the method is that the air region is not meshed, so no numerical noise decreases the accuracy of the stray field computation. The method has already shown good results in shielding effects computation or magnetic signature of ferromagnetic hulls. A combination of FEM and moment method can lead also to interesting results [13]. This method seems easier to implement than the use of infinite modeling tools in FEM or coupling with BEM.

\section{B. Forces and Moments Computations}

The computation of force acting on the ferromagnetic devices can be obtained with the following equation [6]:

$$
\mathbf{F}=\sum_{\mathrm{i}=1}^{\mathrm{N}} \iint_{\mathrm{Si}} \mathbf{B}_{\text {ext }}\left(\mathbf{M}_{\mathbf{i}} \cdot \mathbf{n}_{\mathbf{i}}\right) \mathrm{dS}
$$

where $\mathbf{B}_{\text {ext }}$ is the induction created by all the devices (inductor, magnets, and ferromagnetic regions) except the ferromagnetic region considered. Moments can easily be computed too. The method leads to accurate and very fast computations for devices composed with magnets and with a high leakage field (magnetic micro-actuator for example), for which a high density of mesh is needed in FEM modelings. Let us notice that the use of Maxwell's tensor integration on a surface surrounding the region can reduce the computation time significantly.

\section{SOME NumERICAL EXAMPLES}

\section{A. Modeling of an Actuator}

In this section, we present a classical application of magnetostatic moment method. Let us consider an actuator. It is composed by two ferromagnetic cylindrical columns carrying two inductor coils. Between these two columns, another one, with a rectangular section, is composed by a permanent magnet and ferromagnetic material. A bottom support and a top pallet

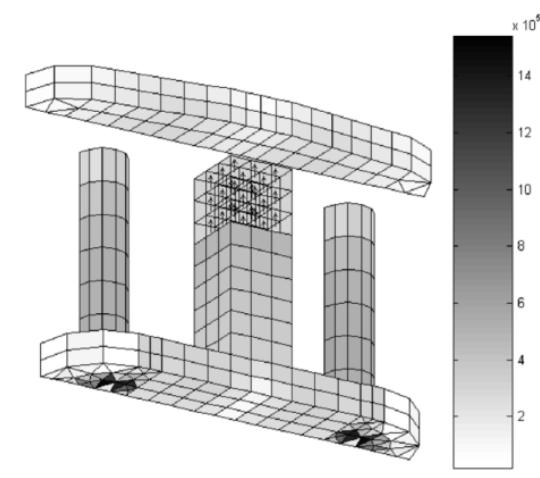

Fig. 4. Magnetization distribution $(\mathrm{A} / \mathrm{m})$ obtained with a standard mesh. Moments directions are not represented $\left(\mu_{\mathrm{r}}=2000\right)$.

channel the induction. The goal of this section is to compute the force acting on the pallet without any current in coils. Let us notice that the device has a symmetry plan. The problem is solved with the 3-D commercial software Flux3D. The force obtained is $25.24 \mathrm{~N}$.

The actuator is now modeled by moment method. The first step is to mesh the active ferromagnetic parts and the magnet by volume elements. A standard FEM mesh generator is used. It is usual to use mapped elements (hexahedral and prism elements). In fact, in the magnetostatic moment method relevant elements must be used. Their shape must be chosen to ensure that the flux will be well-channeled. Indeed, tetrahedral elements often lead to numerical difficulties (bad condition number of the matrix obtained). With such a mapped mesh, the integration is made and the problem solved. The force is computed thanks to (24). It is equal to $22.88 \mathrm{~N}$. The method provides an error of $10 \%$.

Even if the error provided by MoM is not so bad (less than $10 \%$ with few elements), the result cannot be considered as satisfying and must be improved. In fact, this result has mainly two causes. The first one deals with the mesh at the interface between cylindrical columns and the bottom support. As we can see in Fig. 4, some prism elements have a very high magnetization in comparison with their neighbors. A numerical problem seems to occur there. In fact, in this area, the flux makes a strong direction change $\left(90^{\circ}\right)$. As we have already said, the shape of elements must help the flux to have a good direction and prism element, in this device, does not. A simple solution consists in changing both cylindrical columns in equivalent ones with the same square sections. In fact, the reluctance of the circuit will be not modified (nor the flux), and the force will be the same. This result has been verified with FEM modeling. With this assumption, only hexahedral elements can be used. However, this numerical trick is not sufficient, because another main problem occurs. Let us remember that the magnetic field (and so the force) is calculated thanks to equivalent charge distribution. In fact, charges are a representation of the flux [see (13)]. A good way to apply moment method is to imagine where these charges are going to have the highest variation and then to refine the mesh in this area and not elsewhere. It allows to keep an acceptable number of elements. In our device, the permeability is high, thus induction flux is well-channeled by the ferromagnetic material. Charges on most part of interfaces between elements are going to cancel themselves (the external normal are in opposite directions) except where the flux goes through the columns to join 


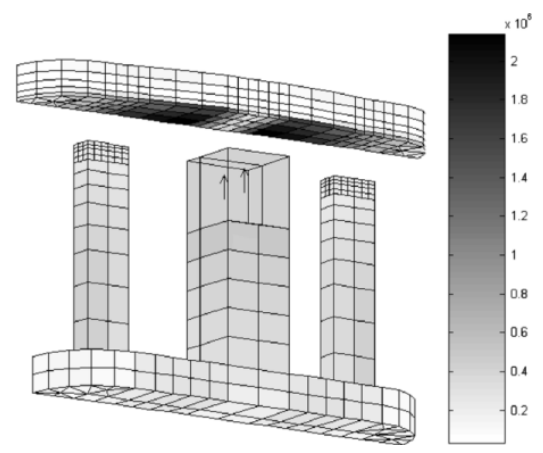

Fig. 5. Magnetization distribution (A/m) obtained with an adapted mesh.

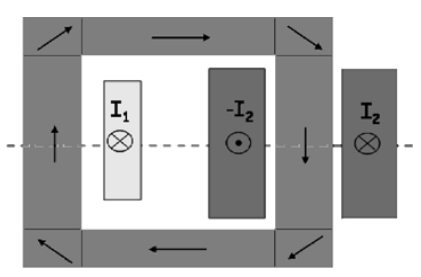

Fig. 6. Mesh of a currents transformer with eight volume elements. Only seven unknowns are necessary to describe the problem (symmetries).

the pallet (air gap of the actuator). It is thus a good solution to refine the mesh there (top of the two square equivalent columns and bottom of the pallet). Let us notice that the magnetization of magnet being known, no mesh refinement is needed in this region. The mesh and magnetizations obtained are presented in Fig. 5. Let us notice also that nonconformal mesh is used. This is one of the main advantages of the method in comparison with FEM where the use of nonconformal mesh is not obvious. The computed force is equal to $25.04 \mathrm{~N}$ (less than $1 \%$ in comparison with FEM). This problem is a good example of moment method use to model devices with complex magnetic circuit geometries.

\section{B. Simplified Moment Method}

This section deals with the study of a current transformer which generates consequent magnetic leakage due to saturation. The goal is to find an electromagnetic model as light as possible (few unknowns so very fast resolution) to allow a global optimization strategy (coupling with electronic supply). The chosen method is the magnetostatic MoM used with very few elements, in which some directions for magnetizations are imposed (see Fig. 6). This approach will be called "simplified moment method."

Simultaneous resolutions of (9) with a nonlinear material law (see Section II-C2) make it possible for a couple of current currents $\left(\mathrm{I}_{1}, \mathrm{I}_{2}\right)$ to determine magnetic state of the device. Then, we can deduce the flux under the secondary coil. A response surface of this flux can thus be built. Each time step, $\mathrm{I}_{2}$ is obtained reading $\mathrm{I}_{2}\left(\mathrm{I}_{1}, \Phi_{2}\right)$. Due to physical relation connecting current transformer variables, $\mathrm{I}_{2}$ can be deduced from $\mathrm{I}_{1}$. Let us notice that the use of "light" moment method leads to an acceptable response surface time computation.

This approach is reliable and accurate while the magnetization can be considered as constant in each element (this is the case for high saturation level in the magnetic circuit). However, for low current values, magnetizations will be not uniform. According to our own experience, to refine the mesh in the current

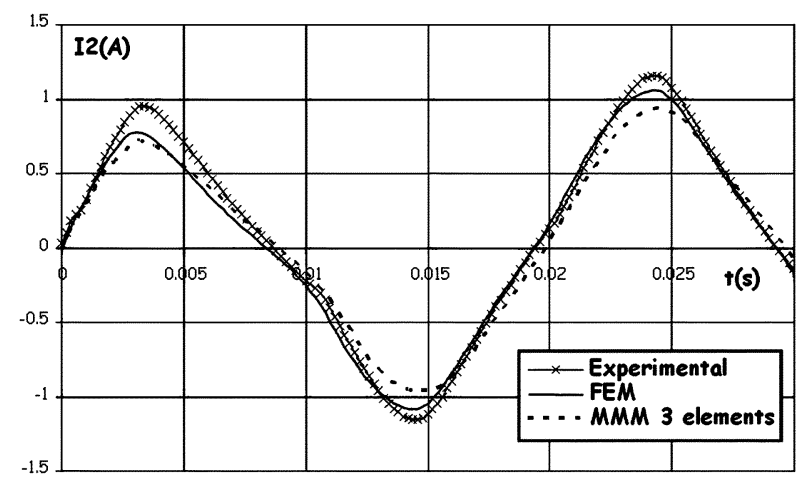

Fig. 7. Comparison with the results obtained by MoM, FEM, and measurements for the currents transformer. Computation times of MMM are divided per 10 in comparison of FEM.

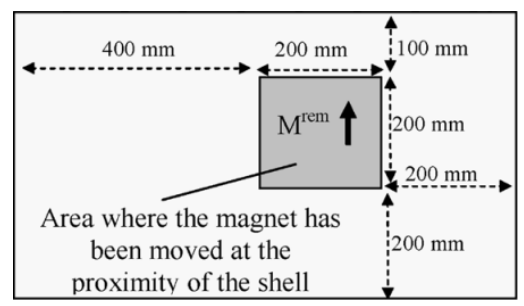

Fig. 8. Localization and direction of the magnetization anomaly created by the SmCo magnet.

transformer could lead to accurate results but this solution is too time consuming to build the response surface.

To improve the results at low currents, the main idea is to combine moment method with reluctance network method, well-known for its accuracy in linear cases. This approach is equivalent to restore the Ampere's law in the matrix and vector coefficient of (9) and is explained in [14]. The combination of two very simple models (a reluctance network and a MoM) for two different cases of operation modes (saturated and nonsaturated) can thus lead to accurate modeling of complex devices. Fig. 7 shows results obtained with this approach and compares them with classical FEM and measurements.

\section{Moment Method in Inverse Problems}

Another interesting numerical application of magnetostatic moment method deals with magnetization identification. This approach is an inverse problem, i.e., the determination of the sources (magnetizations) by knowing the effect (the magnetic field measured on a limited number of a sensor around the device). The problem is the following. We consider a ferromagnetic sheet placed in the earth magnetic field $\mathbf{H}_{0}$. This sheet has an unknown permanent magnetization. In order to increase it and to be more explicit, we create a strong magnetic anomaly in the thin plate with a SmCo permanent magnet (see Fig. 8).

Equations governing the problem are similar to those obtained in (11). The only difference is that each element has unknown magnetizations $m_{\text {ind }}$ and $m_{\text {rem. }}$. By rewriting (11) and by noticing that in this case $\{\mathbf{G}\}=\{\mathbf{F}\}$, we obtain the following system:

$$
\left\{\left\{\mathbf{I}_{\mathbf{d}}\right\}-\{\mathbf{F}\}\right\}\left[\mathbf{m}_{\text {ind }}\right]-\{\mathbf{F}\}\left[\mathbf{m}_{\text {rem }}\right]=\left[\mathbf{h}_{\mathbf{0}}\right] .
$$

This system is not square. It has twice more unknowns than equations. It solution is thus not unique. We have thus to add 


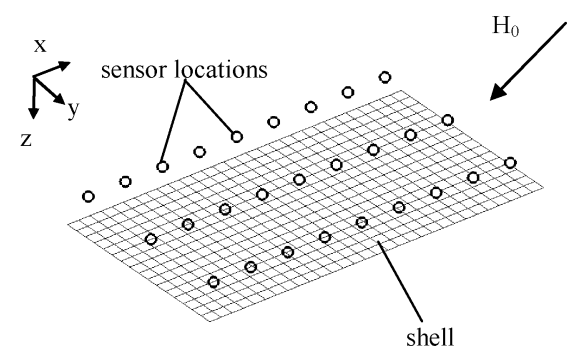

Fig. 9. Ferromagnetic sheet $900 \times 500 \times 1.4 \mathrm{~mm}, \mu_{\mathrm{r}}=96$ with mesh and sensor locations.

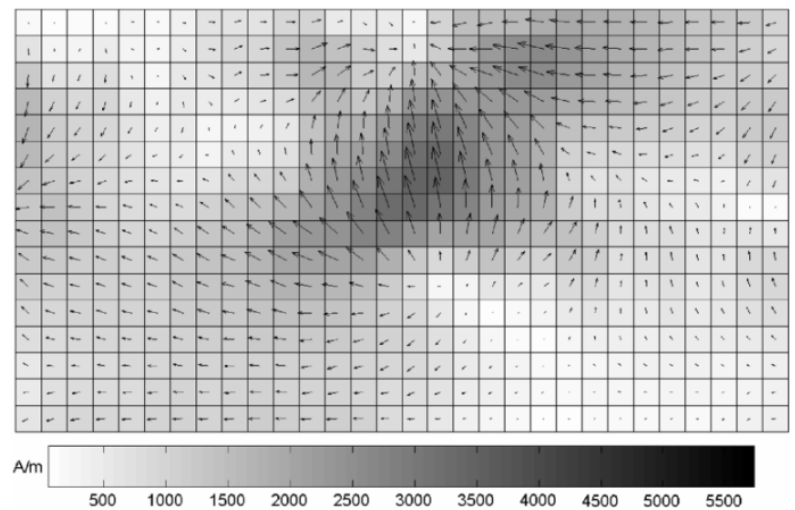

Fig. 10. Identification of the total magnetization of the sheet obtained $(\mathrm{A} / \mathrm{m})$.

measurements information. Twenty-seven tri-axis sensors are placed above the ferromagnetic sheet (see Fig. 9).

The relation linking the measured field to the unknown magnetizations can easily be expressed thanks to (9), the matching point being the sensor location and $\mathbf{h}_{\text {mes }}$ being the measured magnetic field

$$
\{\mathbf{A}\}\left[\mathbf{m}_{\text {ind }}\right]-\{\mathbf{A}\}\left[\mathbf{m}_{\text {rem }}\right]=\left[\mathbf{h}_{\text {mes }}-\mathbf{h}_{\mathbf{0}}\right] .
$$

It remains to solve both system (25) and (26) in the same time with a pseudo-inverse resolution. Then, we get the unknown magnetizations. Many others details about the method are given in [15].

\section{CONCLUSION}

In this paper, we have shown different potentialities of the magnetostatic moment method. Based on a point matching approach of the material law, this numerical method is easy to implement and lead to small matrix systems and therefore to fast resolutions. The approach has demonstrated its ability to accurately solve problems with consequent magnetic leakage (permanent-magnet structures like microsytems, magnetic shieldings, etc.). However, for devices with complex magnetic circuit, a good knowledge of the numerical approach is needed to avoid high mesh density which leads to an unacceptable increase of the memory space.
Moreover, according to us, one of the main advantages of the method is to allow inverse modeling easier than with traditional FEM. Because the models are very light, optimization strategies and identifications can be advantageously provided.

All the numerical examples showed in this paper have been solved with a software package implementing the magnetic moment method and called LOCAPI.

\section{ACKNOWLEDGMENT}

This work was supported in part by Schneider Electric and the French Navy.

\section{REFERENCES}

[1] R. F. Harrington, Field Computation by Moment Methods. New York: IEEE Press, 1993.

[2] M. J. Newman, C. W. Trowbridge, and L. R. Turner, "GFUN: An interactive program as an aid to magnet design," in Proc. 4th Int. Conf. Magnet Technology, Brookhaven National Laboratory, Upton, NY, 1972, pp. 617-626.

[3] O. Chubar, P. Elleaume, and J. Chavanne, "A three-dimensional magnetostatic computer code for insertion devices," J. Synchrotron Rad., vol. 5, pp. 481-484, 1998.

[4] M. Souza, C. Vidigal, A. Momy, J. Taquin, and M. Sauzade, "Non linear calculation of 3D static magnetic fields," IEEE Trans. Magn., vol. 33, no. 4, pp. 2486-2491, Jul. 1997.

[5] O. Chadebec, J.-L. Coulomb, G. Cauffet, J.-P. Bongiraud, and S. Guerin, "Magnetization identification problem-Illustration of an effective approach," COMPEL, vol. 23, no. 2, pp. 518-530, 2004.

[6] E. Durand, Magnétostatique. Paris, France: Masson et Cie, 1968.

[7] P. Elleaume, O. Chubar, and J. Chavanne, "Computing 3D magnetic fields form insertion devices," in Particle Accelerator Conf., vol. 3, 1997, pp. 3509-3511.

[8] H. Zijlstra, Experimental Method in Magnetism-2. Measurement of Magnetic Quantity. Amsterdam, The Netherlands: North-Holland, 1967.

[9] O.-M. Kwon, C. Surussavadee, M. V. K. Chari, S. Salon, and K. K. Sivasubramaniam, "Analysis of the far field of permanent-magnet motors and effects of geometric asymmetries and unbalance in magnet design," IEEE Trans. Magn., vol. 40, no. 2, pp. 435-442, Mar. 2004.

[10] O. Chadebec, J.-L. Coulomb, J. P. Bongiraud, G. Cauffet, and P. Le Thiec, "Recent improvement for solving inverse magnetostatic problem applied to thin shells," IEEE Trans. Magn., vol. 38, no. 2, pp. 1005-1008, Mar. 2002.

[11] S. Guerin, J.-L. Coulomb, G. Cauffet, and L.-L. Rouve, "Analytic integration for closed-loop degaussing computation," in Proc. Int. Conf. Marine Electromagnetic, London, U.K., Mar. 2004.

[12] O. Chadebec, L.-L. Rouve, and J.-L. Coulomb, "New method for a fast and easy computation of stray fields created by wound rods," IEEE Trans. Magn., vol. 38, no. 2, pp. 517-520, Mar. 2002.

[13] B. Froidurot, L.-L. Rouve, A. Foggia, J.-P. Bongiraud, and G. Meunier, "Magnetic discretion of naval propulsion machines," IEEE Trans. Magn., vol. 38, no. 2, pp. 1185-1188, Mar. 2002.

[14] F. Janet, J.-L. Coulomb, C. Chillet, and P. Mas, "Simplified magnetic moment method applied to current transformer modeling," IEEE Trans. Magn., vol. 40, no. 2, pp. 818-821, Mar. 2004.

[15] O. Chadebec, J.-L. Coulomb, V. Leconte, J. P. Bongiraud, and G. Cauffet, "Modeling of static magnetic anomaly created by iron plates," IEEE Trans. Magn., vol. 36, no. 4, pp. 667-671, Jul. 2000.

Manuscript received June 27, 2005 (e-mail: olivier.chadebec@leg.ensieg. inpg.fr). 